\title{
Use of endoscopic ultrasound in pre-treatment staging of esophageal cancer did not alter management plan
}

\author{
Mark Radlinski, Linda W. Martin, Dustin M. Walters, Patrick Northup, Andrew Y. Wang, Terri Rodee, \\ Bryan G. Sauer, Vanessa M. Shami \\ Department of Gastroenterology and Hepatology, University of Virginia, Charlottesville, VA, USA \\ Contributions: (I) Conception and design: M Radlinski, VM Shami; (II) Administrative support: M Radlinski, VM Shami; (III) Provision of study \\ materials or patients: M Radlinski, VM Shami; (IV) Collection and assembly of data: M Radlinski, VM Shami; (V) Data analysis and interpretation: \\ All authors; (VI) Manuscript writing: All authors; (VII) Final approval of manuscript: All authors. \\ Correspondence to: Vanessa M. Shami, MD. Department of Gastroenterology and Hepatology, University of Virginia, 1240 Jefferson Park Ave, \\ Charlottesville, VA 22903, USA. Email: VMS4E@hscmail.mcc.virginia.edu.
}

Background: Initial staging of esophageal cancer relies on EUS in addition to FDG-PET/CT. It is our hypothesis that with the advancement of FDG-PET/CT staging, endoscopic ultrasound may not be required for initial staging in all cases. The purpose of this study is to analyze whether EUS affects initial treatment stratification in patients diagnosed with esophageal cancer.

Methods: A retrospective database at the University of Virginia was queried for patients diagnosed with esophageal squamous cell carcinoma and adenocarcinoma who underwent EGD with EUS and FDG-PET/ CT at their initial evaluation from $10 / 2013$ to 5/2017. Two thoracic surgeons were asked to determine appropriate management for each case. Options included surgical resection, neoadjuvant chemoradiotherapy followed by resection, definitive chemoradiotherapy, or chemotherapy with or without palliative radiation. Both surgeons received the FDG-PET/CT report along with the EGD report. For each case, one or both surgeons were randomly allocated to review EUS results in addition to the clinical information. The treatment decisions of each thoracic surgeon were compared to determine if EUS reports impacted clinical management. Simple and weighted correlation coefficients (kappa) were calculated to compare agreement of treatment choices between the two surgeons using McNemars test. Conditional logistic regression was used to assess the influence of EUS on the treatment recommendations.

Results: A total of 50 patients (44 male and 6 female) were enrolled and data was collected. The thoracic surgeons agreed on treatment decisions in 39 cases and disagreed on 11 cases. Agreement between surgeons was good despite lack of EUS information for one surgeon on each case (weighted Kappa =0.73, 95\% CI: 0.57-0.89). Using conditional logistic regression, EUS did not have a statistically independent association with agreement on treatment plan (P for model $=0.17$ ).

Conclusions: EUS did not have a statistically independent association with agreement on treatment plan for newly diagnosed esophageal cancer ( $\mathrm{P}$ for model $=0.17$ ). Our findings suggest that EUS may not be necessary in the algorithm for the initial staging of every case of esophageal cancer. Selective, rather than mandatory use of EUS seems warranted.

Keywords: Esophageal cancer; staging; endoscopic ultrasound

Submitted Mar 13, 2020. Accepted for publication Aug 14, 2020.

doi: $10.21037 /$ jtd-20-1299

View this article at: http://dx.doi.org/10.21037/jtd-20-1299 


\section{Introduction}

Esophageal cancer is currently the eighth most common cancer worldwide $(1,2)$. Adenocarcinoma arising from Barrett's esophagus is the most common type in the US; with an incidence of 2.8 per 100,000 in 2012 (3) and rising. Esophageal cancer is staged using TNM with depth of tumor invasion ( $\mathrm{T}$ stage), lymphatic dissemination ( $\mathrm{N}$ stage) of malignant esophageal cells, and presence of distant metastases (M stage). Surgeons and oncologists determine treatment modalities (surgical resection, chemotherapy, and/or radiation) and timing of therapies based on TNM staging. Currently, EUS is used in addition (4) to FDGPET/CT for staging esophageal cancer. PET has been previously shown to be superior to a combination of EUS and CT in the diagnosis of metastatic disease (5-7). However, PET has been shown to have low accuracy in staging locoregional disease (8) and EUS has been shown to be superior to PET and CT in T-staging and equivalent in nodal staging (9) While FDG-PET was previously found to rarely affect treatment decisions by thoracic surgeons in esophageal cancer management (10), advances in FDGPET have improved the staging accuracy of this modality. At the same time, the usefulness of routine EUS has been called into question (11). We therefore hypothesize that in the current era of FDG-PET/CT staging, EUS adds little value to FDG-PET and endoscopy. The purpose of this study is to specifically analyze whether EUS affects surgeons' initial treatment stratification in patients diagnosed with esophageal cancer. We present the following article in accordance with the MDAR and STROBE reporting checklist (available at http://dx.doi.org/10.21037/ jtd-20-1299).

\section{Methods}

The charts of fifty patients (44 male and 6 female) at the University of Virginia Health System with primary esophageal cancer were retrospectively reviewed from 2013 through 2017 (Table 1). Each patient had been enrolled into an IRB (\#15945) approved database at the time of initial diagnosis. Each of the patients had biopsy proven esophageal adenocarcinoma or squamous cell carcinoma. Each patient underwent EGD with EUS, and FDG-PET/CT as part of our institution's standard workup. A single dedicated ultrasonographer (VMS), who performs more than 400 EUS procedures per year, performed all staging EUS procedures. Two American Board of Thoracic Surgery certified thoracic surgeons, who annually perform more than thirty esophageal resections for cancer, reviewed presented clinical data to decide on treatment allocation. Each surgeon was provided with patient characteristics including age, gender, and presence or absence of dysphagia. Additionally, each were given EGD and PET/CT reports, while also blinded to patient identifiers. We then randomized the availability of EUS information so that each surgeon saw the EUS reports for only half of all the given cases. The surgeons then chose one of the following treatment strategies: surgical resection, neoadjuvant chemoradiotherapy followed by resection, definitive chemoradiation, or chemotherapy with or without palliative radiation. The treatment decisions were compared to determine whether EUS altered clinical management. Further, to evaluate for inter- and intraobserver variability, five random cases were repeated in each surgeon's review list. Coefficients of agreement $(\mathrm{K})$ were calculated between the treatment decisions. The trial was conducted in accordance with the Declaration of Helsinki (as revised in 2013). The study was approved by institutional review board of the University of Virginia. Per IRB protocol, patients did not give informed consent as this was a retrospective chart review trial.

\section{Imaging modalities/techniques}

Every patient underwent upper endoscopy prior to EUS using a diagnostic scope (Olympus GIF-160 and Olympus GIF-180; Olympus America Inc., Melville, New York, USA). The Olympus GF-UE160 was used to perform the conventional radial echoendoscopic examinations and the Olympus GF-UC140P or GF-UCT180 echoendoscopes were used to perform linear echoendoscopy. For exams requiring fine needle aspiration (FNA), a 22-gauge needle (Wilson-Cook Inc., Winston-Salem, NC) was used. The seventh edition of the AJCC staging guidelines was used to stage the esophageal cancers (12). Lesions that were limited to the lamina propria or muscularis mucosae were labeled as T1a and lesions that invaded the submucosa were labeled as T1b. T2 lesions were defined as tumor invading into the muscularis propria. T3 tumors were defined as those invading into the adventitia. T4 cancers were defined as tumors invading adjacent structures (T4a invading pleura, pericardium, or diaphragm; and T4b invading other structures such as aorta, vertebral body, or the trachea). $\mathrm{T}$ stage and the presence or absence of both local and distant lymph nodes as well as liver metastasis were systematically evaluated for in each patient. 
Table 1 Patient characteristics

\begin{tabular}{lc}
\hline Characteristics & Values \\
\hline Average age, years & 64.94 \\
Sex & \\
Male & $44(88 \%)$ \\
Female & $6(12 \%)$ \\
Dysphagia & $40(80 \%)$ \\
Presenting T stage & \\
T1 & $11(22 \%)$ \\
T2 & $5(10 \%)$ \\
T3 & $33(66 \%)$ \\
T4 & $1(2 \%)$ \\
Ethnicity & \\
White/Caucasian & $44(88 \%)$ \\
African American & $3(6 \%)$ \\
Hispanic & $1(2 \%)$ \\
Asian & $1(2 \%)$ \\
Other & $1(2 \%)$ \\
\hline
\end{tabular}

Since even healthy patients may have visible mediastinal lymph nodes especially in the subcarina, established EUS criteria (hypoechoic, distinct borders, size) were used to distinguish benign from malignant lymph nodes. This permitted the ultrasonographer to target the more suspicious nodes for FNA. When cancer was suspected in a lymph node or liver, sampling was performed with a curvilinear array echoendoscope without passing through the primary tumor. Either a 22 -gauge or 25 -gauge needle was used, and one to three passes were typically performed into the target lesion. The sample was interpreted by an experienced onsite cytopathologist. The majority of FDG-PET/CT studies were performed at UVA. At UVA, FDG-PET/CT (Siemens Biograph 40, Hoffman Estates, IL) without IV contrast (usually with oral contrast) was performed 60 minutes after IV administration of $15 \mathrm{mCi}$ F-18 FDG. All patients had fasted at least six hours prior to injection. Blood glucose levels were checked prior to each procedure (goal $<150 \mathrm{mg} / \mathrm{dL}$ ). The authors are accountable for all aspects of the work in ensuring that questions related to the accuracy or integrity of any part of the work are appropriately investigated and resolved.

\section{Statistical analysis}

Data analysis and manipulation were performed with SAS (version 9.4, Cary, NC). Simple and weighted correlation coefficients (kappa) were calculated to compare choice of treatment between the two observers using McNemar's test. Multivariable logistic regression using the method of maximum likelihood estimates was used to assess the independent influence of EUS on the treatment recommendations. Intraobserver variability between the two observers was measured with blinded reassessments of sample cases again using kappa calculations. The level of statistical significance for type 1 error was set at $\mathrm{P}<0.05$. All significance testing was two-sided.

\section{Results}

Fifty patients ( 44 male and 6 female), mean ages of 65 years old (range, 38-82) with primary esophageal cancer were included in this study. Thirty-nine patients (78\%) had adenocarcinoma and $11(22 \%)$ had squamous cell carcinoma of the esophagus. EUS was performed in all 50 (100\%) cases. There were no complications of EUS or EUS-FNA in any of the 50 cases. No patients were excluded from the analysis. Of the 50 cases analyzed, the thoracic surgeons agreed on treatment decisions in 39 cases and disagreed on 11 cases. Agreement between observers was good (Weighted Kappa $=0.73$, 95\% CI: 0.57-0.89). Using conditional logistic regression, EUS did not have a statistically independent association with agreement on treatment plan ( $\mathrm{P}$ for model $=0.17$ ).

\section{Discussion}

The epidemiology of esophageal cancer in the United States has changed over the past three decades, shifting from a predominance of squamous cell carcinoma to adenocarcinoma (13). Over that time, diagnostic and staging modalities have also substantially evolved. Accurate staging based on depth of tumor invasion and presence of nodal and/or distant metastasis, is the most critical aspect when determining the optimal treatment plan for patients. Currently, the National Comprehensive Cancer Network (NCCN) recommends EUS and CT scan for invasive esophageal cancer, and PET-CT if there is no overt evidence of metastases.

Endoscopic ultrasound is currently standard of care in assessment of locoregional staging of esophageal cancer 
$(14,15)$. However, the impact of EUS on medical decisionmaking is less clear. EUS is utilized to assess for evidence of deep infiltration for accurate $\mathrm{T}$ staging and can also assist in locoregional lymph node evaluation. EUS assessment has previously been found to be diagnostically accurate for $\mathrm{T}$ staging in $71 \%$ overall (16) patients and $87 \%$ with lesions $>\mathrm{T} 2$ (17). For patients without distant metastases, a clear understanding of the $\mathrm{T}$ and $\mathrm{N}$ stage (locoregional stage) is an important factor when deciding between treatment strategies such as endoscopic resection, surgery alone, or neoadjuvant chemoradiation followed by surgery, for instance. NCCN staging guidelines recommend neoadjuvant chemoradiation for locally advanced, at least $\mathrm{T} 3$ and/or N1 esophageal cancer.

Esophagectomy alone can be considered for node negative T1a lesions, T1b, and in some cases of T2 lesions. Previously, EUS provided additional utility in staging by identifying celiac lymph node involvement not identified by PET-CT as EUS can help differentiate between tumor and adjacent lymph nodes (PET-CT superior in identifying distant metastasis). Identification of positive celiac lymph nodes was important because prior to 2010 their presence staged patients as having M1a disease. This is the likely reason that earlier studies such as Preston et al. and Gheorge et al. found that EUS staging altered management in patients with newly diagnosed esophageal cancer $(18,19)$. Now with the change from the AJCC sixth TNM classification to the seventh, where celiac lymph node involvement is now considered regional node metastasis $(\mathrm{N})$ and no longer distant metastatic (M1a) (20), the utility of EUS staging has become more limited. A more recent study by Findlay et al. found that the benefit of EUS might be outweighed by procedural risk (21).

Another consideration is that the majority, $82 \%$, of the patients in this study, presented with dysphagia on initial presentation. A recent study looked at a cohort of one hundred forty-seven patients who presented with dysphagia (68\% of population), of which 133 (90.5\%) had a partially or completely obstructing mass present on endoscopy. For those patients with dysphagia and a partially or fully obstructing mass, it was found that the proportion of patients with locally advanced disease was significantly increased $(\mathrm{P}<0.001)$ as compared to patients without dysphagia or without a mass. Dysphagia and an obstructing mass had a sensitivity of 0.89 and 0.88 specificity for (at least) locally advanced disease (22). Patients with both dysphagia and an esophageal mass most commonly receive neoadjuvant chemoradiation with surgery, definitive chemoradiation, or palliative therapy. Another study showed dysphagia was present in $89 \%$ of patients with T3-T4 disease by EUS and only $53 \%$ of patients without dysphagia had T3T4 disease by EUS $(\mathrm{P}<0.001)$ (23). EUS is less likely to affect treatment decisions in patients with dysphagia, as this is a sensitive and specific surrogate marker for transmural disease.

Our study found that EUS did not have a statistically independent association with agreement on treatment plan for newly diagnosed esophageal cancer ( $\mathrm{P}$ for model $=0.17$ ). Of those 11 cases (Table 2) where the thoracic surgeons disagreed, five of them had the same exact information (both had access to EUS reports). We believe the difference is secondary to surgeon preference on management. Of the six cases where one of the surgeons was blinded to EUS while the other was not, there was not a consistent pattern to how this affected the treatment decision. Also, the group size was likely too small to accurately identify any differences. Unfortunately, we were not able to glean any meaningful insight from these cases with discrepant treatment decisions. Our findings question the necessity of EUS in the algorithm for the initial staging of esophageal cancer. This likely is in the setting of a diminished role for celiac lymph node evaluation in the differentiation between local and metastatic disease. Additionally, in the literature, dysphagia appears to be at least an equal predictor of $\mathrm{T} 3$ or greater disease than EUS. The utility for EUS may increase if reserved for patients without dysphagia. Another scenario where EUS guided tissue acquisition is helpful is targeting distant lymph nodes or liver metastases noted on cross sectional imaging or during a carefully performed staging EUS exam. These findings would most likely put the patient in the nonoperative category.

This study is limited due to a relatively small (50 total patients) sample size. Additionally, junctional cancers were not included in this study. While, in our study, performance of EUS did not globally affect surgeon agreement for treatment plan, further studies are needed to evaluate the impact of EUS in esophageal cancer staging. This study suggests that there may be a limited role for EUS in the initial staging of esophageal cancer in patients who have undergone EGD and FDG-PET. At our institution, the professional fee charged for EUS is $\$ 582.00$ whereas EUS/ FNA is $\$ 1,045.00$. This is a significant expense for patients that could be potentially avoided if EUS and EUS/FNA is not needed for staging. Given that EUS is less likely to affect treatment decisions as mentioned above, a future 
Table 2 Eleven discrepant cases

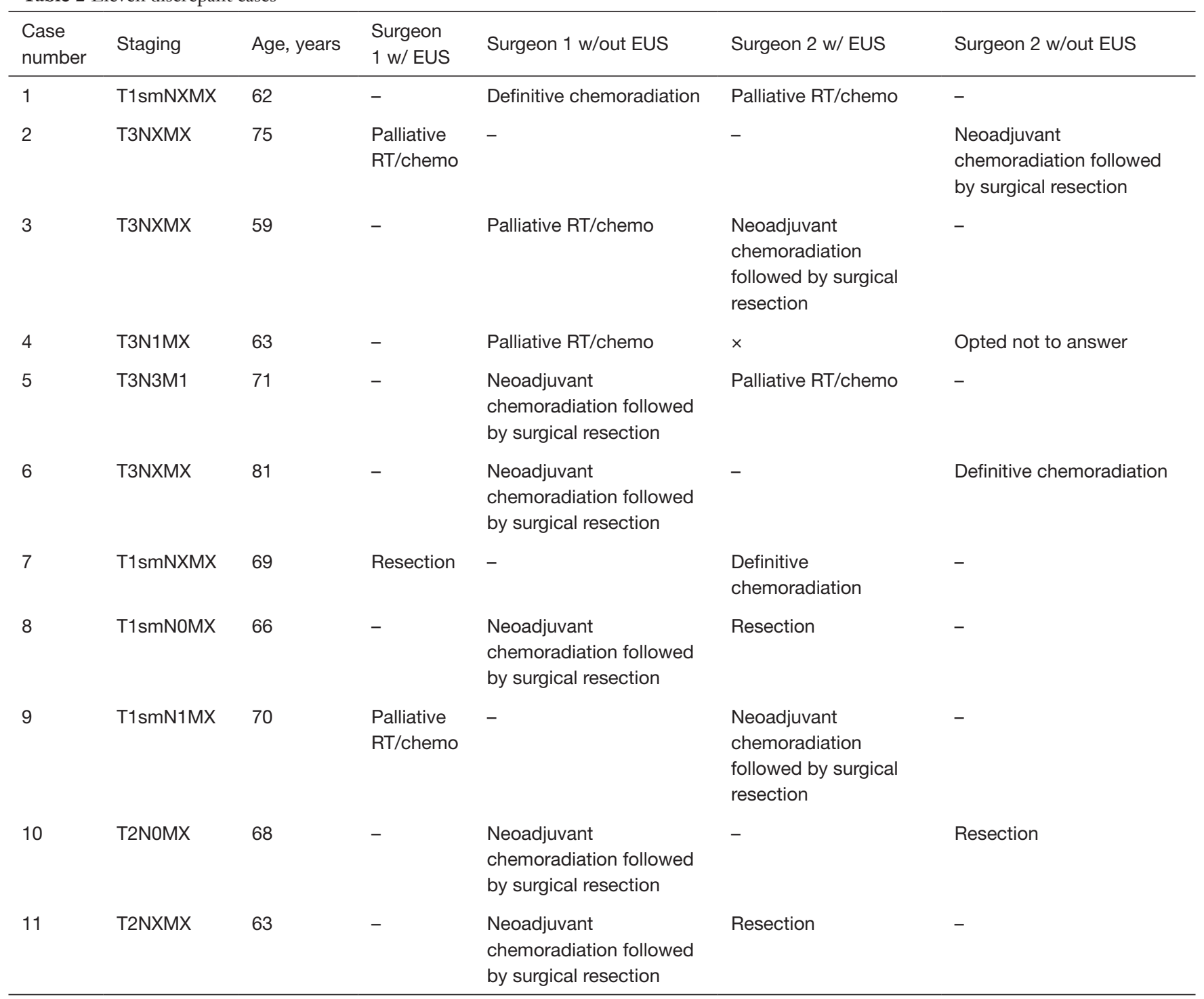

study would be useful to determine if EUS may have utility as part of the algorithm for staging in patients who do not initially present with dysphagia.

\section{Conclusions}

In conclusion, use of endoscopic ultrasound in pretreatment staging of esophageal cancer did not alter the management plan. An algorithm for the initial staging of esophageal cancer should consist of EGD and FDG-PET as primary modalities with EUS serving as an adjunct for patients without dysphagia upon presentation.

\section{Acknowledgments}

Funding: None.

\section{Footnote}

Reporting Checklist: The authors have completed the MDAR and STROBE reporting checklist. Available at http://dx.doi. org/10.21037/jtd-20-1299

Data Sharing Statement: Available at http://dx.doi. org/10.21037/jtd-20-1299 
Conflicts of Interest: All authors have completed the ICMJE uniform disclosure form (available at http://dx.doi. org/10.21037/jtd-20-1299). LWM reports Consultant for Pacira Pharmaceuticals, Astra Zeneca, Steering Committee member for ELUCIDATE trial for On Target Laboratories; VMS reports personal fees from Olympus America, personal fees from Interpace Diagnostics, outside the submitted work. The authors have no other conflicts of interest to declare.

Ethical Statement: The authors are accountable for all aspects of the work in ensuring that questions related to the accuracy or integrity of any part of the work are appropriately investigated and resolved. The trial was conducted in accordance with the Declaration of Helsinki (as revised in 2013). The study was approved by institutional review board of the University of Virginia. Per IRB protocol, patients did not give informed consent as this was a retrospective chart review trial.

Open Access Statement: This is an Open Access article distributed in accordance with the Creative Commons Attribution-NonCommercial-NoDerivs 4.0 International License (CC BY-NC-ND 4.0), which permits the noncommercial replication and distribution of the article with the strict proviso that no changes or edits are made and the original work is properly cited (including links to both the formal publication through the relevant DOI and the license). See: https://creativecommons.org/licenses/by-nc-nd/4.0/.

\section{References}

1. DeSantis CE, Lin CC, Mariotto AB, et al. Cancer treatment and survivorship statistics, 2014. CA Cancer J Clin 2014;64:252-71.

2. Rustgi AK, El-serag HB. Esophageal carcinoma. N Engl J Med 2014;371:2499-509.

3. Ferlay J, Soerjomatram M, Ervik R, et al. Globocan 2012 v1.1, Cancer Incidence and mortality worldwide: IARC CancerBase No.11. Available online: https://publications. iarc.fr/Databases/Iarc-Cancerbases/GLOBOCAN-2012Estimated-Cancer-Incidence-Mortality-And-PrevalenceWorldwide-In-2012-V1.0-2012

4. National Comprehensive Cancer Network. NCCN clinical practice guidelines in oncology: esophageal and esophagogastric junction cancers. 2017. Available online: https://www.nccn.org/professionals/physician_gls/default.asp

5. Flamen P, Lerut A, Van Cutsem E, et al. Utility of positron emission tomography for the staging of patients with potentially operable esophageal carcinoma. J Clin Oncol 2000;18:3202-10.

6. Flamen P, Van Cutsem E, Lerut A, et al. Positron emission tomography for assessment of the response to induction radiochemotherapy in locally advanced oesophageal cancer. Ann Oncol 2002;13:361-8.

7. Yoon YC, Lee KS, Shim YM, et al. Metastasis to regional lymph nodes in patients with esophageal squamous cell carcinoma: CT versus FDG PET for presurgical detection prospective study. Radiology 2003;227:764-70.

8. Rice TW. Clinical staging of esophageal carcinoma. CT, EUS, and PET. Chest Surg Clin N Am 2000;10:471-85.

9. Lowe VJ, Booya F, Fletcher JG, et al. Comparison of positron emission tomography, computed tomography, and endoscopic ultrasound in the initial staging of patients with esophageal cancer. Mol Imaging Biol, 2005;7:422-30.

10. McDonough PB, Jones DR, Shen KR, et al. Does FDGPET add information to EUS and CT in the initial management of esophageal cancer? A prospective single center study. Am J Gastroenterol 2008;103:570-4.

11. DaVee T, Ajani JA, Lee JH. Is endoscopic ultrasound examination necessary in the management of esophageal cancer? World J Gastroenterol 2017;23:751-62.

12. Rice TW, Blackstone EH, Rusch VW. 7th edition of the AJCC Cancer Staging Manual: esophagus and esophagogastric junction. Ann Surg Oncol 2010;17:1721-4.

13. Brown LM, Devesa SS, Chow WH. Incidence of adenocarcinoma of the esophagus among white Americans by sex, stage, and age. J Natl Cancer Inst 2008;100:1184-7.

14. Wallace MB, Nietert PJ, Earle C, et al. An analysis of multiple staging management strategies for carcinoma of the esophagus: computed tomography, endoscopic ultrasound, positron emission tomography, and thoracoscopy/laparoscopy. Ann Thorac Surg 2002;74:1026-32.

15. Puli SR, Reddy JB, Bechtold ML, et al. Staging accuracy of esophageal cancer by endoscopic ultrasound: a metaanalysis and systematic review. World J Gastroenterol 2008; $14: 1479$.

16. O'Farrell NJ, Malik V, Donohoe CL, et al. Appraisal of staging endoscopic ultrasonography in a modern high-volume esophageal program. World J Surg 2013;37:1666-72.

17. Rice TW, Blackstone EH, Adelstein DJ, et al. Role of clinically determined depth of tumor invasion in the treatment of esophageal carcinoma. J Thorac Cardiovasc Surg 2003;125:1091-102. 
18. Preston SR, Clark GW, Martin IG, et al. Effect of endoscopic ultrasonography on the management of 100 consecutive patients with oesophageal and junctional carcinoma. Br J Surg. 2003;90:1220-4.

19. Gheorghe C, Stanescu C, Gheorghe L, et al. Preoperative noninvasive EUS evaluation in patients with esophageal cancer considered for esophagectomy. J Gastrointestin Liver Dis 2006;15:137-41.

20. Ajani JA, D'Amico TA, Almhanna K, et al. Esophageal and esophagogastric junction cancers, version 1.2015. J Natl Compr Canc Netw 2015;13:194-227.

21. Findlay JM, Bradley KM, Maile EJ, et al. Pragmatic

Cite this article as: Radlinski M, Martin LW, Walters DM, Northup P, Wang AY, Rodee T, Sauer BG, Shami VM. Use of endoscopic ultrasound in pre-treatment staging of esophageal cancer did not alter management plan. J Thorac Dis 2020;12(10):5850-5856. doi: 10.21037/jtd-20-1299 staging of oesophageal cancer using decision theory involving selective endoscopic ultrasonography, PET and laparoscopy. Br J Surg 2015;102:1488-99.

22. Mansfield SA, El-Dika S, Krishna SG, et al. Routine staging with endoscopic ultrasound in patients with obstructing esophageal cancer and dysphagia rarely impacts treatment decisions. Surg Endosc 2017;31:3227-33.

23. Ripley RT, Sarkaria IS, Grosser R, et al. Pretreatment Dysphagia in Esophageal Cancer Patients May Eliminate the Need for Staging by Endoscopic Ultrasonography. Ann Thorac Surg 2016;101:226-30. 